\title{
Polpa do cacau (Theobroma cacao L.) como substrato na elaboração de bebidas funcionais potencialmente probióticas
}

\author{
Cocoa pulp (Theobroma cacao L.) as a substrate in the preparation of potentially probiotic \\ Pulpa de cacao (Theobroma cacao L.) como sustrato en la preparación de bebidas funcionales \\ potencialmente probióticas
}

Recebido: 02/08/2021 | Revisado: 10/08/2021 | Aceito: 19/08/2021 | Publicado: 21/08/2021

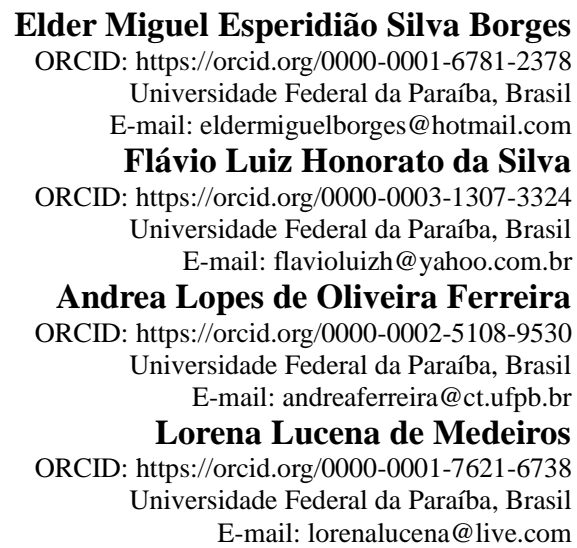

\begin{abstract}
Resumo
O cacau (Theobroma cacao L.), fruto do cacaueiro, é o principal ingrediente presente na composição do chocolate. Porém para a sua produção, é utilizado somente as sementes, tornando a polpa deste fruto um resíduo agroindustrial. Há muitos estudos que relataram alguns produtos a partir dessa polpa, como geleias, bebidas finas e até mesmo sorvetes. Baseado nessas aplicações da polpa do cacau, o presente estudo teve por objetivo realizar a caracterização físico-química da polpa do cacau, e avaliar seu potencial para a produção de bebidas potencialmente probióticas. Inicialmente foi realizada a caracterização da polpa, através da análise do pH, acidez titulável (ATT), teor de sólidos solúveis (SST), açúcares redutores (AR) e açúcares redutores totais (ART). Durante a análise, verificou-se que, com exceção do teor de sólidos solúveis, todos os parâmetros estavam dentro do estabelecido pela legislação vigente e de acordo com os dados obtidos a partir da revisão de literatura. Após uma revisão sistemática e a análise dos dados obtidos, foi possível verificar que a polpa do cacau possui enorme potencial para ser aplicada em processos de obtenção de bebidas potencialmente probióticas. O teor de sólidos solúveis e a concentração de açúcares geram maior impacto nos processos de obtenção dessa bebida, tendo em vista que são os componentes de maior consumo durante o processo.
\end{abstract}

Palavras-chave: Probióticos; Cacau; Suco de frutas; Alimentos funcionais.

\begin{abstract}
Cocoa (Theobroma cacao L.), a fruit of the cocoa tree, is the main ingredient present in the composition of chocolate. However, for its production, the seeds are the only part used, making the pulp of this fruit an agro-industrial residue. Basically, this species can be categorized into three varieties, Forastero, Criollo and Trinitário, each one being responsible for unique characteristics that end up influencing the final product after processing. Many studies have obtained products from this pulp, such as jellies, fine drinks and even ice cream. Based on these applications of cocoa pulp, this study aimed to carry out the physicochemical characterization of cocoa pulp and to evaluate its potential for the production of potentially probiotic beverages. Initially, the characterization of the pulp was carried out through the analysis of $\mathrm{pH}$, titratable acidity (ATT), soluble solids content (SST), reducing sugars (AR) and total reducing sugars (ART). During the analysis, it could be noticed that, with the exception of the soluble solids content, all parameters were within the established by current legislation and in accordance with the data obtained from the literature review. After the literature review and the analysis of the obtained data, it was possible to verify that the cocoa pulp has enormous potential to be applied in processes intended to obtain potentially probiotic beverages. The soluble solids content and the concentration of sugars have the greatest impact on the process of obtaining this drink, considering that they are the most consumed components during the process.
\end{abstract}

Keywords: Probiotics; Cocoa; Fruit juice; Functional foods. 


\begin{abstract}
Resumen
El cacao (Theobroma cacao L.), fruto del árbol del cacao, es el principal ingrediente presente en la composición del chocolate. Sin embargo, para su producción solo se utilizan las semillas, por lo que la pulpa de esta fruta es un residuo agroindustrial. Básicamente, esta especie se puede dividir en tres variedades, Forastero, Criollo y Trinitário, cada una responsable de unas características únicas que acaban influyendo en el producto final tras su elaboración. Son muchos los estudios que reportan algunos productos de esta pulpa, como jaleas, refrescos e incluso helados. Con base en estas aplicaciones de la pulpa de cacao, el presente estudio tuvo como objetivo realizar la caracterización físico-química de la pulpa de cacao y evaluar su potencial para la producción de bebidas potencialmente probióticas. Inicialmente, la caracterización de la pulpa se realizó mediante el análisis de pH, acidez titulable (TT), contenido de sólidos solubles (SST), azúcares reductores (AR) y azúcares reductores totales (ART). Durante el análisis, se encontró que, a excepción del contenido de sólidos solubles, todos los parámetros se encontraban dentro de lo establecido por la legislación vigente y de acuerdo con los datos obtenidos de la revisión de la literatura. Luego de un relevamiento bibliográfico y análisis de los datos obtenidos, se pudo constatar que la pulpa de cacao tiene un enorme potencial para ser aplicada en procesos de obtención de bebidas potencialmente probióticas. El contenido de sólidos solubles y la concentración de azúcares tienen un mayor impacto en los procesos de obtención de esta bebida, considerando que son los componentes más consumidos durante el proceso.
\end{abstract}

Palabras clave: Probióticos; Cacao; Zumo de frutas; Alimentos funcionales.

\title{
1. Introdução
}

O cacau (Theobroma cacao L.) endêmico da região da Bacia do Amazonas, já é produzido em diversos continentes, como América, África, Ásia e Oceania. No final da década de 90, as plantações cacaueiras do nordeste da Bahia, sofreram a incidência do fungo Crinipellis perniviosa, conhecido por "vassoura de bruxa", onde o mesmo devastou a produção de cacau no país, reduzindo em aproximadamente 53\%. Porém, mesmo com essa redução e problemática, o Brasil ainda se destacou no cenário mundial, ocupando o lugar de quarto maior produtor do mundo (Veríssimo, 2012). Após o último levantamento realizado, em 2019. O Brasil ocupa o sétimo lugar no ranking dos maiores países produtores de cacau no mundo, com 265.000 toneladas, o equivalente a 4,6\% (Brainer, 2021).

A espécie Theobroma cacao L. pode gerar três variedades distintas de fruto: o tipo Criollo, o tipo Forastero e o tipo Trinitário, onde cada um possui características específicas e que os diferem um do outro (Icco, 2012; Cruz, 2002). O fruto do cacau tipo Criollo, possui aparência alongada com pontas proeminentes, superfície externa rugosa, sementes ovais e soltas em sua polpa mucilaginosa. Chama a atenção pelo seu sabor e aroma ameno devido à ausência de antocianinas responsáveis pelo sabor forte e adstringente, e devido à essas características é considerado uma variedade mais refinada (Cruz, 2002). Em contrapartida, essa variedade é muito mais suscetível a doenças e devido a essa característica, representa uma pequena porcentagem de toda a produção mundial de cacau (Sequeira, 2016). Já o cacau do tipo Forastero, apresenta fruto no formato mais redondo, casca rígida e livre de rugas. Suas sementes são mais compactadas e se dispõem presas em sua polpa. Apresenta sabor e aroma bem mais pronunciado e amargo devido à presença das antocianinas, e por isso não é muito bem aceito pela indústria alimentícia. Entretanto, essa variedade é altamente resistente a condições climáticas severas o que a torna responsável pela maior produção mundial, aproximadamente 95\% (Cruz, 2002; Sequeira, 2016). Por fim, o cacau do tipo Trinitário, que nada mais é que o resultado do cruzamento entre o Criollo e o Forastero, apresenta características bioquímicas dos dois grupos e é considerado um híbrido de qualidade superior aos demais (Cruz, 2002).

A polpa do cacau, devido ao seu alto volume, acaba se tornando um resíduo agroindustrial e a busca por sua aplicabilidade vem crescendo de forma considerável (Almeida \& Valle, 2007). A partir de alguns estudos, foi possível observar o uso da polpa do cacau para diversos produtos, como: geleias de cacau, bebidas finas e fermentadas, álcool, vinagre, iogurtes, sorvetes, sucos e até mesmo a polpa em sua forma natural (Babatope, 2005). Outro resíduo considerável na indústria cacaueira é a casca do cacau, uma vez que compõe aproximadamente 70\% do fruto (Donkoh et al., 2008). Apesar de ser considerado um resíduo, a polpa do cacau é uma matéria-prima com grande potencial de aplicabilidade na indústria (Almeida \& Valle, 2007), sendo sua composição formada por água (aproximadamente 85\%), açúcares (12,5\%) e outros compostos em 
quantidades reduzidas, tais como proteínas e ácido cítrico (Soares, 2001). A composição química do cacau está diretamente ligada a inúmeros fatores, onde os principais são o tipo de cacau, a origem das amêndoas, o manejo ambiental e especialmente o quão maduro estão os frutos.

A busca por um novo estilo de vida tem tornado os consumidores mais dispostos a seguirem uma rotina alimentar mais saudável. Atrelado a esse estilo de vida, houve uma crescente procura por alimentos que não somente sirva como fonte de nutrientes, mas que também, tragam benefícios adicionais a saúde, como a diminuição de doenças (Fontes, 2013; Renuka et al., 2009).

Para que um alimento seja considerado funcional, o mesmo precisa atuar de forma benéfica em uma ou mais função no organismo, ou seja, além de desempenhar o papel de fornecer nutrientes, o mesmo precisa, de algum modo, promover de forma relevante a saúde e o bem-estar (Moraes \& Colla, 2006; Roberfroid, 2002).

No Brasil, o órgão que regulamenta os alimentos funcionais é a ANVISA (Agência Nacional de Vigilância Sanitária), que tornou público em 30 de abril de 1999 três resoluções acerca do tema. A resolução de ${ }^{\circ} 17$ discute e aprova as diretrizes responsáveis para avaliar a segurança dos alimentos; a resolução de $\mathrm{n}^{\circ} 18$ aprova as diretrizes referentes as análises e a comprovação de suas propriedades funcionais tomando por base a rotulagem de cada alimento e a resolução de ${ }^{\circ} 19$ aprova os procedimentos para o registro final dos alimentos funcionais (Brasil, 1999a; Brasil, 1999b; Brasil, 1999c).

Uma das formas de se produzir e comercializar alimentos funcionais, é através dos alimentos probióticos. Essa classe de alimentos ganhou força no cenário da alimentação saudável, e cada vez mais vem atraindo um público maior.

Probióticos são microrganismos vivos, que quando ingeridos em quantidades adequadas conferem benefícios à saúde do hospedeiro por meio do desenvolvimento da flora microbiana no intestino (Saad, 2006). Podem ser encontrados pela literatura com outros termos como, bioterapêuticos, bioprofiláticos e bioprotetores (Reig \& Anesto, 2002).

Há alguns fatores que interferem na permanência desses microrganismos no intestino, como pH, concentração de substrato e a interação bacteriana. Assim sendo, os microrganismos pertencentes aos gêneros Bifidobacterium e Lactobacillus são os de maior interesse industrial para produção de alimentados fermentados (Coelho, 2009).

Os probióticos são encontrados no comércio de diversas formas, seja como alimentos, bebidas, suplementos e até mesmo como medicamentos. Entretanto, esses alimentos estão diretamente relacionados a produtos lácteos, o que de certa forma gera algumas limitações aos consumidores (Pereira et al., 2017).

A utilização de suco de frutas e outros produtos vegetais visando a substituição do leite, vem ganhando espaço cada vez maior no ramo alimentício. Essa crescente se dá devido ao fato de que uma série de benefícios estão associados a esses alimentos, como a ausência de colesterol e a presença de vitaminas, minerais, fibras e compostos antioxidantes (Pereira et al., 2011).

São muitas as frutas aplicadas como fonte de produtos probióticos. Em seus estudos, Amorim et al. (2010), avaliaram o potencial do suco do abacaxi como matriz em um processo fermentativo, e obtiveram resultados que mostraram a capacidade probiótica dessa matéria-prima, sugerindo que o suco de fruta avaliado é um bom substrato para a agregação de culturas probióticas.

Com isso, o presente artigo teve por objetivo caracterizar a polpa do cacau e avaliar sua aplicação como potencial substrato na produção de bebidas probióticas e utilizar a meta-análise como artifício para a comparação de dados da literatura.

\section{Metodologia}

O trabalho é apresentado em duas etapas. Primeira etapa utilizou-se da pesquisa de natureza qualitativa e a segunda etapa de natureza qualitativa (uma revisão sistemática). Todo o trabalho da etapa quantitativa foi realizado no Laboratório de 
Bioengenharia, no Centro de Tecnologia, na Universidade Federal da Paraíba, João Pessoa - Paraíba. Para o preparo dos sucos, foram utilizadas polpas industrializadas da marca GUTTI®, lote 0544 com validade até 25 de fevereiro de 2021 , as quais foram armazenadas congeladas $\left(-18^{\circ} \mathrm{C}\right)$ até o uso. Para preparo dos sucos, a polpa foi diluída na proporção de $100 \mathrm{~g}$ de polpa para $200 \mathrm{~mL}$ de água.

A polpa foi inicialmente caracterizada quanto ao seu $\mathrm{pH}$ por potenciometria direta. $\mathrm{O}$ phmetro foi calibrado com soluções tampão de pH 7,0 e 4.0, seguindo a metodologia descrita por AOAC (1994).

A acidez foi determinada pela titulação da amostra com solução de $\mathrm{NaOH}$ a $0,1 \mathrm{~N}$, e utilizou-se, como indicador do ponto de viragem, a solução alcoólica de fenoftaleína a 1\%, de acordo com a metodologia descrita por IAL (2008).

Para a determinação do 0Brix, foi utilizado o refratômetro de Abbé digital, onde foi inserido $1 \mathrm{~mL}$ da amostra no equipamento, e procedeu-se com a leitura. O resultado foi expresso de forma corrigida de acordo com a temperatura (IAL, 2008).

Para a determinação dos teores de açúcares redutores e açucares redutores totais, foi utilizado a metodologia descrita por Miller (1959), através da reação de redução do ácido 3,5-dinitrosalicílico a 3-amino-5-nitrosalicílico (DNS).

Após a obtenção dos dados, primeira etapa do trabalho, a partir da caracterização físico-química, foi realizada, segunda etapa do trabalho, uma análise dos dados, a fim de comparar os resultados obtidos com o que há de mais recente na literatura e também realizado um levantamento de trabalhos, revisão sistemática (Callou et al., 2021) que objetivaram a produção de produtos probióticos a partir de diferentes matrizes, sendo a principal delas o suco de frutas. Em seguida, foram comparados esses parâmetros entre os resultados obtidos pelos diferentes autores. Após a apresentação do comparativo entre os dados obtidos com trabalhos recentes, foi realizado uma análise entre outros trabalhos que buscaram realizar a produção de bebidas probióticas utilizando algumas matrizes como fonte de substrato. Foi feito um levantamento de 120 trabalhos onde a principal característica entre eles, foi a utilização de frutas ou parte de frutas para a caracterização e/ou síntese de algum produto. Após o levantamento, aplicou-se uma filtragem dos trabalhos selecionados, os de maior interesse para a discussão. Após essa seleção, 08 trabalhos foram selecionados na meta-análise para a discussão dos dados obtidos a partir dos resultados. Dos 08 trabalhos analisados, 04 verificaram alguns parâmetros da polpa do cacau.

\section{Resultados e Discussão}

\subsection{Caracterização físico-química}

A Tabela 1, apresenta os resultados obtidos a partir das análises físico-químicas da polpa do cacau.

Tabela 1 - Resultados obtidos a partir das análises físico-químicas para a polpa do cacau.

\begin{tabular}{ccc}
\hline Análise & Resultado & Valor mínimo exigido* \\
\hline $\mathrm{pH}$ & $3,46 \pm 0,02$ & 3,4 \\
Acidez Titulável & $1,18 \pm 0,05 \mathrm{de} \%$ ácido cítrico & $0,75 \%$ de ácido cítrico \\
Sólidos Solúveis Totais & $11,07 \pm 0,12{ }^{\circ} \mathrm{Brix}$ & $14{ }^{\circ} \mathrm{Brix}$ \\
Açúcares Redutores & $9,01 \pm 9,26 \mathrm{~g} / 100 \mathrm{~g}$ & - \\
Açúcares Redutores Totais & $11,51 \pm 4,97 \mathrm{~g} / 100 \mathrm{~g}$ & $10 \mathrm{~g} / 100 \mathrm{~g}$ \\
\hline
\end{tabular}

*Valores exigidos pela legislação de acordo com a Instrução Normativa $\mathrm{n}^{\mathrm{o}} 37$ de $1^{\mathrm{o}}$ de outubro de 2018 , fixada pelo Ministério da Agricultura, Pecuária e Abastecimento (Brasil, 2018).

Fonte: Autores (2021).

Após a análise do pH da polpa do cacau, foi possível identificar o valor de 3,46 $\pm 0,02$, o que vai de acordo com trabalhos realizados por Penha e Matta (1998), onde observaram algumas características físico-químicas da polpa do cacau.

Todos os resultados encontrados após as análises estão de acordo com o que está estabelecido pela legislação vigente, 
com exceção do teor de sólidos solúveis, que apresentou, em média de valor, $20 \%$ menor ao exigido pela Instrução Normativa $\mathrm{n}^{\circ} 37$ de $1^{\circ}$ de outubro de 2018, fixada pelo Ministério da Agricultura, Pecuária e Abastecimento (Brasil, 2018).

Alguns fatores podem influenciar diretamente o $\mathrm{pH}$ da polpa do cacau, sendo o principal deles a presença de alguns ácidos orgânicos, como ácido acético e o ácido cítrico. Em estudos realizados, foi possível identificar o pH de 3,75 ligeiramente superior ao encontrado neste trabalho. Anvoh et al. (2009), associam esse comportamento ao fato de que esses ácidos, presentes na polpa, desempenham funções de preservação antibacteriana do produto. Essas características químicas, refletem diretamente no grau de preservação desse alimento, uma vez que a propagação de microrganismos em meios ácidos é mais difícil de ocorrer, favorecendo assim a conservação.

Tendo em vista os parâmetros analisados, o pH da polpa do cacau apresentou um valor bastante satisfatório tendo em vista a sua aplicação como fonte para a produção de produtos probióticos. Seu comportamento em um processo fermentativo se caracteriza como um dos fatores de maior interferência no processo e influencia diretamente na fermentação.

Através da análise de acidez titulável, foi obtido um valor de 1,18\% de ácido cítrico. Esse dado mostra que a faixa de acidez na polpa do cacau é ligeiramente menor que a acidez do fruto in natura. Porém, esse dado da acidez está de acordo com os parâmetros estabelecidos pela Instrução Normativa n 37 de $1^{\circ}$ de outubro de 2018, fixada pelo Ministério da Agricultura, Pecuária e Abastecimento, onde diz que o valor mínimo de acidez aceitável é de 0,75\% de ácido cítrico (Brasil, 2018).

Em trabalhos realizados por Alexandre et al. (2015), onde analisaram diversas características de alguns clones de cacau, obtiveram valores para acidez numa faixa entre 1,57 até $2,12 \%$ de ácido cítrico.

Há alguns estudos que mostram essa pequena diferença na acidez de polpas de frutas congeladas e frutas in natura. Martim et al. (2013), ao analisarem a acidez de frutos do cupuaçu in natura (espécie pertencente ao gênero Theobroma) encontraram valores em torno de 1,94 \% de ácido cítrico. Já nos resultados encontrados por Freire et al. (2009), onde analisaram polpas de cupuaçu de marcas comerciais, encontraram valores de 1,38\% de ácido cítrico.

Os resultados obtidos neste trabalho estão de acordo com os dados da literatura. Alexandre et al. (2015), ao caracterizar frutos de alguns clones do cacaueiro, identificaram que houve uma pequena variação entre os resultados encontrados na análise da acidez, onde o menor valor encontrado foi de 1,57\% de ácido cítrico e o maior de 2,12\% de ácido cítrico. Esse dado está diretamente relacionado com as características gustativas da polpa do cacau e também com sua preservação diante de microrganismos contaminantes (Alexandre et al., 2015).

O valor obtido para o teor de sólidos solúveis totais (SST) foi de 11,07 ${ }^{\circ}$ Brix, valor considerado bom para polpas de frutas congeladas. Moreira (2017), ao analisar o teor de sólidos solúveis ( ${ }^{\circ}$ Brix) em diversos clones de cacau in natura, obteve uma média de 17,8, valor bem acima do encontrado neste trabalho, o que corrobora com os dados obtidos por Amorim et al. (2010), que ao avaliarem o teor de sólidos solúveis em diferentes marcas de polpas congeladas de cacau, obtiveram valores entre 17 e 19 Brix.

Alguns fatores podem interferir no teor de sólidos solúveis em polpas de frutas, como teor de precipitação, nutrição do solo e a quantidade excessiva de água (Santos, et al., 2002). Alguns outros fatores são mencionados por Honorato et al. (2015), como a intensidade do período de chuvas, a safra em que esses produtos foram cultivados e coletados, e também se houve ou não a adição de água durante o processamento dessa matéria-prima, o que pode levar a diluição dos açúcares presentes, em virtude da adição de água.

Os valores encontrados para os açúcares redutores e açúcares redutores totais foram de 9,01 g/100g e 11,51 g/100g, respectivamente. Em seus estudos, Barreto et al. (2013) investigaram algumas características físico-químicas de polpas do cacau, e verificaram que o valor obtido para os açúcares redutores totais foi de 16,82 g/100g. Pereira et al. (2019), também buscaram identificar as características físico-químicas de clones do cacau, e ao verificarem os teores de açúcares, verificaram valores de 10,3 g/100g para açúcares redutores e 16,0 g/100g para açúcares redutores totais. 
Assim como nos demais parâmetros avaliados neste trabalho, os teores de açúcares podem variar de acordo com vários fatores, sendo os principais: os fatores edafoclimáticos - precipitação e condições do solo e a variação genética do fruto em que a polpa foi extraída, tudo isso acaba resultando em diferentes teores de sólidos de açúcares.

Pereira et al. (2019), avaliaram diferentes clones de cacau coletados em diferentes safras, e pôde verificar que tanto a diferença genética como as diferentes condições de cada safra, interferiram no teor de açúcares. Ao analisar os açúcares redutores da variedade Parazinho coletada em dezembro/2018, foi possível identificar o teor de açúcares de 10,3 g/100g, enquanto a variedade PS1319 coletada em agosto/2018, apresentou o teor de 11,5 g/100g.

\subsection{Avaliação dos dados obtidos por meio de revisão sistemática}

$\mathrm{O}$ primeiro parâmetro analisado durante a revisão sistemática foi o $\mathrm{pH}$. Foi verificado alguns trabalhos onde objetivaram fazer o levantamento dos parâmetros físico-químicos de algumas frutas e todos eles avaliaram esse parâmetro.

A Tabela 2 mostra os dados extraídos durante o processo de meta-análise, e pode-se verificar que todos os autores avaliaram os parâmetros de $\mathrm{pH}$ e teor de sólidos solúveis.

Pereira et al. (2019), buscaram caracterizar três diferentes clones de cacau, sendo eles o Parazinho, o PS1319 e o LP06. Esses autores obtiveram o mesmo valor médio do pH para as três variedades, chegando ao valor de 3,90 $\pm 0,00$. Essa similaridade pode ter-se dado devido ao fato das três variedades terem sido coletadas no mesmo período, ou seja, características edafoclimáticas podem ter gerado essa semelhança nesse parâmetro para os três clones.

Alexandre et al. (2015) também buscaram caracterizar a polpa de diferentes variedades de cacau, e para o pH, obtiveram o valor médio de 3,32 $\pm 0,11$, corroborando com o valor médio obtido por Pereira et al. (2019). 
Tabela 2 - Dados obtidos a partir da análise para a variável pH e teor de sólidos solúveis totais.

\begin{tabular}{|c|c|c|c|c|c|}
\hline Autor & Matéria-prima & pH & $\begin{array}{c}\text { pH } \\
\text { médio }\end{array}$ & $\begin{array}{c}\text { SST } \\
\left({ }^{\circ} \text { brix }\right)\end{array}$ & $\begin{array}{c}\text { SST } \\
\text { médio }\end{array}$ \\
\hline \multirow{6}{*}{ Honorato et al. (2015) } & Maracujá & 3,23 & \multirow{6}{*}{$3,55 \pm 0,55$} & 9,32 & \multirow{6}{*}{$11,18 \pm 1,75$} \\
\hline & Abacaxi & 3,92 & & 12,7 & \\
\hline & Caju & 4,13 & & 12 & \\
\hline & Cacau & 3,66 & & 13,39 & \\
\hline & Umbu & 2,61 & & 9,4 & \\
\hline & Graviola & 3,72 & & 10,28 & \\
\hline \multirow{3}{*}{$\begin{array}{l}\text { Pereira et al. } \\
\qquad(2019)\end{array}$} & Cacau parazinho & 3,90 & \multirow{3}{*}{$3,90 \pm 0,00$} & 14,57 & \multirow{3}{*}{$15,56 \pm 0,92$} \\
\hline & Cacau PS1319 & 3,90 & & 15,7 & \\
\hline & Cacau LP06 & 3,90 & & 16,4 & \\
\hline \multirow{10}{*}{$\begin{array}{l}\text { Santos et al. } \\
\text { (2019) }\end{array}$} & Abacaxi & 3,56 & \multirow{10}{*}{$3,98 \pm 1,03$} & 18 & \multirow{10}{*}{$11,40 \pm 3,03$} \\
\hline & Ameixa & 3,07 & & 7 & \\
\hline & Caqui & 5,28 & & 13 & \\
\hline & Laranja & 4,06 & & 11 & \\
\hline & Maçã & 3,88 & & 13 & \\
\hline & Mamão & 4,50 & & 11 & \\
\hline & Maracujá & 2,61 & & 12 & \\
\hline & Melão & 6,00 & & 10 & \\
\hline & Morango & 3,35 & & 8 & \\
\hline & Uva & 3,50 & & 11 & \\
\hline \multirow{7}{*}{ Alexandre et al. (2015) } & Cacau CC10 & 3,19 & \multirow{7}{*}{$3,32 \pm 0,11$} & 16,55 & \multirow{7}{*}{$14,43 \pm 1,23$} \\
\hline & Cacau CEPEC2002 & 3,36 & & 14,35 & \\
\hline & Cacau PH15 & 3,22 & & 12,97 & \\
\hline & Cacau CCN51 & 3,24 & & 15,05 & \\
\hline & Cacau PS1319 & 3,45 & & 15,05 & \\
\hline & Cacau TSH1188 & 3,45 & & 13,65 & \\
\hline & Cacau PH16 & 3,31 & & 13,42 & \\
\hline Penha e Mata & Cacau de safra & 3,26 & \multirow{2}{*}{$3,44 \pm 0,25$} & 17 & \multirow{2}{*}{$17,75 \pm 1,06$} \\
\hline$(1998)$ & Cacau temporão & 3,62 & & 18,5 & \\
\hline Machado e Rizzato (2019) & Maracujá & 4,44 & 4,44 & 2,5 & 2,50 \\
\hline $\begin{array}{c}\text { Ribeiro } \\
(2019)\end{array}$ & Cajá & 6,63 & 6,63 & 7,85 & 7,85 \\
\hline Gonçalves et al. (2013) & Cupuaçu & 3,68 & 3,68 & 13 & 13,00 \\
\hline
\end{tabular}

Fonte: Autores (2011).

Além da análise do $\mathrm{pH}$ desses frutos, esses autores identificaram o teor de sólidos solúveis que estão compondo essa matéria-prima. Como citado anteriormente, esse teor de sólidos, na maioria das vezes compostos por açúcares, é um parâmetro de grande importância a ser avaliado.

Em seu trabalho, Ribeiro (2019) buscou produzir uma bebida probiótica utilizando como substrato a polpa do cajá, utilizando o Lactobacillus acidophilus como microrganismo na fermentação. Após o processo foi verificado o decaimento do teor de sólidos solúveis de 8,0 Brix para 7,85 ${ }^{\circ}$ Brix. Esse decréscimo nesse parâmetro está intimamente relacionado ao consumo de açúcares pelos microrganismos para a sua manutenção e estabilidade na bebida.

Uma forma de confirmar esse comportamento desse parâmetro, seria ter avaliado o teor de açúcares redutores totais no início e no final de todo o processo. Entretanto, a autora não buscou avaliar esse parâmetro para reafirmar o que foi observado no teor de sólidos solúveis.

Machado e Rizato (2019), também objetivaram algo semelhante ao trabalho de Ribeiro (2019). Esses autores buscaram produzir uma bebida probiótica a partir da polpa do maracujá, utilizando o Lactobacillus casei como microrganismo na fermentação. No início do processo, o teor de sólidos solúveis foi ajustado para 3,5 Brix, sendo esse valor reduzido para 2,5 ${ }^{\circ}$ Brix. Essa pequena diminuição pode ter ocorrido devido ao consumo dos açúcares presentes na polpa e consequente produção de ácidos ou utilização do microrganismo para sua manutenção (energia). 
A acidez, outro parâmetro avaliado durante a meta-análise, teve comportamento bastante similar dentro dos frutos da

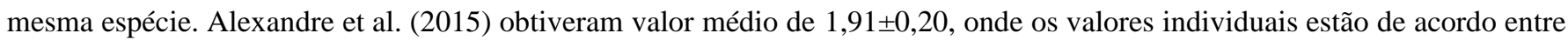
si, devido aos dados verificados pelo desvio.

Alguns frutos da mesma espécie, apresentaram valores bastante divergentes para a acidez. Um deles é foi o maracujá, avaliado por Honorato et al. (2015), apresentando valor de 2,51\% de ácido cítrico, enquanto no trabalho de Santos et al. (2019), apresentou valor de 39,13\% de ácido cítrico.

É preciso levar em consideração alguns pontos para poder compreender essa divergência de valores para tal análise. Essas espécies podem ter sofrido diferentes condições de manejo, assim como podem ter passado por diferentes condições climáticas durante o processo de cultivo. O grau de maturação também precisa ser levado em consideração.

O fruto do maracujá é naturalmente mais ácido que os demais, ou seja, há uma maior concentração de ácidos orgânicos em sua polpa - geralmente ácido cítrico e ácido málico. Quanto maior os níveis desses ácidos, menor o grau de maturação dos frutos, uma vez que o elevado índice de ácidos orgânicos inibe o crescimento de alguns microrganismos responsáveis por acelerar o processo de maturação.

Na Tabela 3, estão os dados obtidos através da meta-análise para o parâmetro da acidez. Os dados estão expressos em $\%$ de ácido cítrico.

Tabela 3 - Dados obtidos a partir da análise para a variável Acidez.

\begin{tabular}{|c|c|c|c|}
\hline Autor & Matéria-prima & Acidez (\% ácido cítrico) & Acidez média \\
\hline Honorato et al. (2015) & $\begin{array}{c}\text { Maracujá } \\
\text { Abacaxi } \\
\text { Caju } \\
\text { Cacau } \\
\text { Umbu } \\
\text { Graviola }\end{array}$ & $\begin{array}{l}2,51 \\
0,57 \\
0,46 \\
0,72 \\
1,65 \\
0,84\end{array}$ & $1,13 \pm 0,80$ \\
\hline Pereira et al. (2019) & $\begin{array}{c}\text { Cacau parazinho } \\
\text { Cacau PS1319 } \\
\text { Cacau LP06 }\end{array}$ & $\begin{array}{l}1,40 \\
1,40 \\
1,20\end{array}$ & $1,33 \pm 0,12$ \\
\hline Santos et al. (2019) & $\begin{array}{c}\text { Abacaxi } \\
\text { Ameixa } \\
\text { Caqui } \\
\text { Laranja } \\
\text { Maçã } \\
\text { Mamão } \\
\text { Maracujá } \\
\text { Melão } \\
\text { Morango } \\
\text { Uva }\end{array}$ & $\begin{array}{c}6,00 \\
11,30 \\
0,08 \\
0,91 \\
1,47 \\
0,20 \\
39,13 \\
0,08 \\
6,13 \\
3,57\end{array}$ & $6,89 \pm 11,90$ \\
\hline Alexandre et al. (2015) & $\begin{array}{c}\text { Cacau CC10 } \\
\text { Cacau CEPEC2002 } \\
\text { Cacau PH15 } \\
\text { Cacau CCN51 } \\
\text { Cacau PS1319 } \\
\text { Cacau TSH1188 } \\
\text { Cacau PH16 }\end{array}$ & $\begin{array}{l}2,12 \\
2,04 \\
2,01 \\
2,04 \\
1,78 \\
1,79 \\
1,57\end{array}$ & $1,91 \pm 0,20$ \\
\hline Penha e Mata (1998) & $\begin{array}{l}\text { Cacau de safra } \\
\text { Cacau temporão }\end{array}$ & $\begin{array}{l}1,55 \\
1,55\end{array}$ & $1,55 \pm 0,00$ \\
\hline Machado e Rizzato (2019) & Maracujá & 6,63 & 6,63 \\
\hline Ribeiro (2019) & Cajá & 0,01 & 0,01 \\
\hline Gonçalves et al (2013) & Cupuaçu & 1,81 & 1,81 \\
\hline
\end{tabular}

Fonte: Autores (2011). 
Um outro parâmetro que vai de acordo com o teor de sólidos solúveis, são os açúcares presentes na matéria-prima. Penha e Mata (1998), caracterizaram polpas de diferentes safras do cacau, e chegaram a valores de 16,6 g/100g e 19,46 g/100g de polpa nas duas safras analisadas. Esses valores vão de acordo com o que foi obtido por Pereira et el. (2019), que obtiveram o valor médio de 11,3 g/100g de polpa. Diante dos trabalhos analisados para a polpa do cacau, essa média dos açúcares está dentro do valor médio obtido por esses autores.

A Tabela 4 descreve os dados de açúcares redutores e açúcares redutores totais para os trabalhos verificados na metaanálise.

Tabela 4 - Dados obtidos a partir da análise para a variável Açúcares Redutores e Açúcares Redutores Totais.

\begin{tabular}{|c|c|c|c|c|c|}
\hline Autor & Matéria-prima & $\begin{array}{c}\mathbf{A R} \\
(\mathrm{g} / \mathbf{1 0 0 g})\end{array}$ & $\begin{array}{c}\text { AR } \\
\text { médio }\end{array}$ & $\operatorname{ART}(\mathrm{g} / \mathbf{1 0 0 g})$ & ART médio \\
\hline \multirow{6}{*}{ Honorato et al. (2015) } & Maracujá & - & \multirow{6}{*}{ 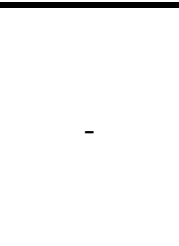 } & - & \multirow{6}{*}{-} \\
\hline & Abacaxi & - & & - & \\
\hline & Caju & - & & - & \\
\hline & Cacau & - & & - & \\
\hline & Umbu & - & & - & \\
\hline & Graviola & - & & - & \\
\hline \multirow{3}{*}{$\begin{array}{l}\text { Pereira et al. } \\
\quad(2019)\end{array}$} & Cacau parazinho & 11,00 & \multirow{3}{*}{$11,30 \pm 0,26$} & 16,55 & \multirow{3}{*}{$16,46 \pm 0,16$} \\
\hline & Cacau PS1319 & 11,50 & & 16,56 & \\
\hline & Cacau LP06 & 11,40 & & 16,28 & \\
\hline \multirow{10}{*}{$\begin{array}{l}\text { Santos et al. } \\
\text { (2019) }\end{array}$} & Abacaxi & 4,61 & \multirow{10}{*}{$6,51 \pm 3,18$} & 16,67 & \multirow{10}{*}{$10,40 \pm 3,83$} \\
\hline & Ameixa & 5,87 & & 7,60 & \\
\hline & Caqui & 12,31 & & 15,82 & \\
\hline & Laranja & 2,38 & & 4,96 & \\
\hline & Maçã & 9,01 & & 9,65 & \\
\hline & Mamão & 5,86 & & 8,29 & \\
\hline & Maracujá & 2,97 & & 6,99 & \\
\hline & Melão & 5,12 & & 10,78 & \\
\hline & Morango & 6,59 & & 9,83 & \\
\hline & Uva & 10,37 & & 13,39 & \\
\hline \multirow{7}{*}{ Alexandre et al. (2015) } & Cacau CC10 & - & \multirow{7}{*}{-} & - & \multirow{7}{*}{-} \\
\hline & Cacau CEPEC2002 & - & & - & \\
\hline & Cacau PH15 & - & & - & \\
\hline & Cacau CCN51 & - & & - & \\
\hline & Cacau PS1319 & - & & - & \\
\hline & Cacau TSH1188 & - & & - & \\
\hline & Cacau PH16 & - & & - & \\
\hline Penha e Mata & Cacau de safra & 8,41 & \multirow{2}{*}{$9,83 \pm 2,00$} & 16,60 & \multirow{2}{*}{$18,03 \pm 2,02$} \\
\hline$(1998)$ & Cacau temporão & 11,24 & & 19,46 & \\
\hline $\begin{array}{c}\text { Machado e Rizzato } \\
\text { (2019) }\end{array}$ & Maracujá & - & - & - & - \\
\hline $\begin{array}{l}\text { Ribeiro } \\
(2019)\end{array}$ & Cajá & - & - & - & - \\
\hline Gonçalves et al. (2013) & Cupuaçu & - & - & - & - \\
\hline
\end{tabular}

Fonte: Autores (2011).

De todas as análises citadas, os teores de açúcares redutores e redutores toais, foram os únicos quantificados apenas por três autores. Para os autores que analisaram o cacau, todos os valores obtidos estão de acordo entre si e obedecendo o que a legislação vigente exige.

Santo et al. (2019), analisaram os teores de açúcares de diferentes frutos. O desvio obtido pela média sofreu bastante variação, devido ao fato de que são frutas muito distintas, e esse teor de açúcares acaba passando por uma oscilação diante da análise. 
Dessa forma, as informações compiladas nas tabelas apresentadas, mostram de forma clara como os resultados das análises realizadas estão de acordo entre si, levando em consideração as particularidades de cada espécie. A aplicação desses produtos em processos que envolvem a produção de bebidas probióticas possui grande potencial, porém é preciso levar em consideração cada ponto analisado e discutidos neste trabalho.

\section{Conclusão}

O presente estudo confirma a polpa de cacau como substrato para a produção de bebidas fermentadas com potencial probiótico. Os resultados obtidos nas caracterizações corroboram com muitos autores onde quantificaram esses parâmetros e até mesmo produziram a bebida probiótica.

Foi demonstrado alguns trabalhos recentes da literatura que estão de acordo com os dados obtidos por meio das análises. E de forma confirmativa, a maior parte dos parâmetros avaliados estão de acordo com a legislação vigente, exceto o parâmetro Sólidos Solúveis, que se apresentou ligeiramente inferior ao mínimo exigido.

Por fim, é importante levar em consideração o único parâmetro analisado que não estava de acordo com a legislação o teor de sólidos solúveis. Alguns fatores podem ter influenciado nesse resultado, onde o principal é o grau de maturação do fruto, uma vez que interfere diretamente nesse parâmetro.

Este artigo buscou apresentar o potencial da polpa do cacau como substrato na elaboração de bebidas probióticas. Em trabalhos futuros, produzir de fato a bebida e avaliar sua aceitabilidade por meio da análise sensorial de alimentos fermentados. Além disso, avaliar o tempo em que o alimento se mantém estável e viável após sua produção.

\section{Referências}

Alexandre, R. S., Chagas, K., Marques, I. P. M., Costa, P. R. \& Filho, J. C. (2015). Caracterização de frutos de clones de cacaueiros na região litorânea de São Mateus, ES. Revista Brasileira de Engenharia Agrícola e Ambiental, 19(8), 785-790.

Almeida, A. A. F. \& Valle, R. R. (2007). Ecophysiology of the cacao tree. Brazilian Journal of Plant Physiology, 19(4), 425-448.

Amorim, G. M., Santos, T. C., Pacheco, C. S. V., Tavares, I. M. C. \& Franco, M. (2010). Avaliação microbiológica, físico-química e sensorial de polpas de frutas comercializadas em Itapetinga - BA. Enciclopédia Biosfera, 7(13), 1391-1398.

Anvoh, K. Y. B., Bi, A. Z. \& Gnakri, D. (2009). Production and characterization of juice from mucilage of cocoa beans and its transformation into marmalade. Pakistan Journal of Nutrition, 8(2), 129-133.

AOAC. (1994). Association of Official Analytical Chemists. Métodos oficiais de análise.

Babatope, B. (2005). Rheology of cocoa-pod husk aqueous system. Part-I: Steady state flow behavior. Rheologica Acta, 45(1), 72-76.

Barreto, F. S., Barreto, W. S., Valle, R. R., Oliveira, J. S., Silva, G. F., Sacramento, C. K., Barreto, L. S. \& Ribeiro, M. A. Q. (2013). Concentração de nutrientes em polpa de cacau produzida no sudeste da Bahia. 53 Congresso Brasileiro de Química - CBQ.

Brainer, M. S. C. P. (2021). Produção de Cacau. Caderno Setorial ETENE.

Brasil. (1999a). Resolução RDC ANVISA n. ${ }^{17}$, de 30 de abril de 1999. Regulamento Técnico que estabelece as Diretrizes Básicas para a Avaliação de Risco e Segurança dos Alimentos.

Brasil. (1999b). Resolução RDC ANVISA n. ${ }^{\circ}$ 18, de 30 de abril de 1999. Regulamento Técnico que estabelece as diretrizes básicas para análise e comprovação de propriedades funcionais e ou de saúde alegadas em rotulagem de alimentos.

Brasil. (1999c). Resolução RDC ANVISA n. ${ }^{\circ}$ 19, de 30 de abril de 1999. Regulamento Técnico de procedimentos para registro de alimento com alegação de propriedades funcionais e ou de saúde em sua rotulagem.

Brasil. (2018). Instrução Normativa n. 97 de 18 de dezembro de 2018. Estabelece os parâmetros analíticos de suco e de polpa de frutas e a listagem das frutas e demais quesitos complementares aos padrões de identidade e qualidade já fixados pelo Ministro da Agricultura, Pecuária e Abastecimento.

Callou, M. A. M., Callou, R. C. M., Coelho, J. L. G., Sampaio, J. R. F., Rocha, E. M. B., Menezes, F. N., Junior, J. G. S., Silva, S. E. T. \& Santana, W. J. (2021). Impacto da intervenção para o consumo de frutas e hortaliças em escolas públicas no Brasil: revisão sistemática com meta-análise e metarregressão, Brazilian Journal of Development. 7(4), 37921-37932.

Coelho, C. J. (2009). Elaboração de bebida probiótica a partir do suco de laranja com Lactbobacillus casei. (Mestre em Ciência e Tecnologia dos Alimentos). Universidade Federal do Ceará, Fortaleza, Ceará. 
Research, Society and Development, v. 10, n. 11, e01101119002, 2021

(CC BY 4.0) | ISSN 2525-3409 | DOI: http://dx.doi.org/10.33448/rsd-v10i11.19002

Cruz, C. L. C. V. (2002). Melhoramento do sabor de amêndoas de cacau através de tratamento térmico em forno convencional e de micro-ondas. (Mestre em Tecnologia de Alimentos). Universidade Estadual de Campinas, Campinas, São Paulo.

Donkoh, A., Atuahene, B. N. \& Adomako, D. (2008). Chemical composition of cocoa pod husk and its effect on growth ond food efficiency in broiler chicks. Animal Feed Science and Technology, 5(10), 1141-1144.

Fontes, C. P. M. L. (2013). Produção de polissacarídeos prebióticos em suco de frutas. (Doutor em Biotecnologia). Universidade Federal do Ceará. Fortaleza, Ceará.

Freire, M. T. A., Petrus, R. R., Freire, C. M. A., Oliveira, C. A. F., Felipe, A. M. P. F. \& Gatti, J. B. (2009). Physical-chemical, microbiological and sensory evaluation of frozen cupuaçu pulp (Theobroma grandiflorum Schum). Brazilian Journal of Food Technology, 12(1), 09-16.

Honorato, A. C., Dias, C. B. R., Souza, E. B., Carvalho, I. R. B. \& Souza, K. S. M. (2015). Parâmetros físico-químicos de polpas produzidas na cidade de Petrolina - PE. Revista Verde de Agroecologia e Desenvolvimento Sustentável, 10(4), 01-05.

IAL. (2008) Normas analíticas do Instituto Adolfo Lutz - Métodos físico-químicos para análises de alimentos. (4a ed.).

ICCO. (2012). Origins Of Cocoa And Its Spread Around The World. 2012. http://www.icco.org/about/growing.aspx.

Machado, L. F. \& Rizzatto, M. L. (2019). Produção e análises físico-químicas de bebida probiótica de suco de maracujá. COGITARE. 2 (1), 50-69.

Moraes, F. P. \& Colla, L. M. (2006). Alimentos Funcionais e Nutracêuticos: definições, legislação e benefícios à saúde. Revista Eletrônica de Farmácia. 3(2), $109-122$.

Penha, E. M. \& Matta, V. M. (1998). Características físico-químicas e microbiológicas da polpa de cacau. EMBRAPA. 33(11), 1945-1949.

Pereira, A. L. F., Feitosa, W. S. C., Abreu, V. K. G., Lemos, T. O., Gomes, W. F. \& Narain, N. (2018). Impact of fermentation conditions on the quality and sensory properties of a probiotic cupuassu (Theobroma grandiflorum) beverage. Food Research International. 100(1), 603-611.

Pereira, A. L. F., Maciel, T. C. \& Rodrigues, S. (2011). Probiotic beverage from cashew Apple juice fermented with Lactobacillus casei. Food Research International. 44(5), 1276-1283.

Pereira, I., Silveira, P. T. S., Martins, M. O. P. \& Efraim, P. (2019). Caracterização da polpa de diferentes cultivares de cacau. XXVIII Congresso de Iniciação Científica da UNICAMP.

Reig, A. L. C. \& Anesto, J. B. (2002). Prebióticos y probióticos, una Relación Beneficiosa. Instituto de Nutrición e Hiene de los Alimentos. Revista Cubana de Alimentação e Nutrição. 16(1), 63-68.

Renuka, B., Kulkarni, S. G., Vijayanand, P. \& Prapulla, S. G. (2009). Fructooligosaccharide fortification of selected fruit juice beverages: effect on the quality characteristics. LWR - Food Science and Technology. 42(5), 1031-1033.

Ribeiro. E. S. S. (2019). Elaboração e caracterização de bebida probiótica a partir do suco de cajá fermentado com Lactobacillus acidophillus NRRL B-4495. (Mestre em Nutrição). Universidade Federal do Rio Grande do Norte. Natal, Rio Grande do Norte.

Roberfroid, M. (2013). Functional food concept and its application to prebiotics. Digestive and Liver Disease. 34(2), 105-110.

Saad, S. M. I. (2006). Probióticos e prebióticos: o estado da arte. Revista Brasileira de Ciências Farmacêuticas. 42(1), 1-16.

Sequeira, A. F. C. (2016). Cacau: do fruto ao chocolate. (Mestre em Biologia). Escola Secundária Rainha Santa Isabel. Estremoz, Portugal.

Soares, M. S. (2001). Estudo do melhoramento do sabor de cacau (Theobroma cacao L.) através de ação enzimática durante a fermentação. (Mestre em Tecnologia de Alimentos). Universidade Estadual de Campinas, Campinas, São Paulo.

Veríssimo, A. J. M. (2012). Efeito da origem do cacau na sua qualidade comercial. Funcional e sensorial. O caso do cacau Catongo de São Tomé e Príncipe e do Brasil. (Mestre em Engenharia Alimentar) Universidade Técnica de Lisboa. Lisboa. 